\title{
Neuroinflammation and Functional Connectivity in Alzheimer's Disease: Interactive Influences on Cognitive Performance
}

\author{
${ }^{\circ}$ L. Passamonti, ${ }^{1,2 *}$ K.A. Tsvetanov, ${ }^{2 *}$ P.S. Jones, ${ }^{2}$ W.R. Bevan-Jones, ${ }^{3}$ R. Arnold, ${ }^{2}$ R.J. Borchert, ${ }^{2}$ E. Mak, ${ }^{3}$ L. Su, ${ }^{3}$ \\ J.T. O'Brien, ${ }^{3 \dagger}$ and $\odot$ J.B. Rowe $2,4^{\dagger}$ \\ ${ }^{1}$ Istituto di Bioimmagini e Fisiologia Molecolare (IBFM), Consiglio Nazionale delle Ricerche (CNR), 20090, Milano, Italy, Departments of ${ }^{2}$ Clinical \\ Neurosciences, ${ }^{3}$ Psychiatry, University of Cambridge, Cambridge CB2 0SZ, United Kingdom, and ${ }^{4}$ Cognition and Brain Sciences Unit, Medical Research \\ Council, Cambridge CB2 7EF, United Kingdom
}

Neuroinflammation is a key part of the etio-pathogenesis of Alzheimer's disease (AD). We tested the relationship between neuroinflammation and the disruption of functional connectivity in large-scale networks, and their joint influence on cognitive impairment. We combined $\left[{ }^{11} \mathrm{C}\right] \mathrm{PK} 11195$ positron emission tomography (PET) and resting-state functional magnetic resonance imaging (rs-fMRI) in 28 patients (12 females/16 males) with clinical diagnosis of probable AD or mild cognitive impairment with positive PET biomarker for amyloid, and 14 age-, sex-, and education-matched healthy controls ( 8 females $/ 6$ males). Source-based "inflammetry" was used to extract principal components of $\left[{ }^{11} \mathrm{C}\right] \mathrm{PK} 11195$ PET signal variance across all participants. rs-fMRI data were preprocessed via independent component analyses to classify neuronal and non-neuronal signals. Multiple linear regression models identified sources of signal covariance between neuroinflammation and brain connectivity profiles, in relation to the diagnostic group (patients, controls) and cognitive status.

Patients showed significantly higher $\left[{ }^{11} \mathrm{C}\right] \mathrm{PK} 11195$ binding relative to controls, in a distributed spatial pattern including the hippocampus, frontal, and inferior temporal cortex. Patients with enhanced loading on this $\left[{ }^{11} \mathrm{C}\right] \mathrm{PK} 11195$ binding distribution displayed diffuse abnormal functional connectivity. The expression of a stronger association between such abnormal connectivity and higher levels of neuroinflammation correlated with worse cognitive deficits.

Our study suggests that neuroinflammation relates to the pathophysiological changes in network function that underlie cognitive deficits in Alzheimer's disease. Neuroinflammation, and its association with functionally-relevant reorganization of brain networks, is proposed as a target for emerging immunotherapeutic strategies aimed at preventing or slowing the emergence of dementia.

Key words: $\left[{ }^{11} \mathrm{C}\right] \mathrm{PK} 11195$; Alzheimer's disease; functional connectivity; independent component analysis; neuroinflammation; PET

Significance Statement

Neuroinflammation is an important aspect of Alzheimer's disease (AD), but it was not known whether the influence of neuroinflammation on brain network function in humans was important for cognitive deficit. Our study provides clear evidence that in vivo neuroinflammation in $\mathrm{AD}$ impairs large-scale network connectivity; and that the link between neuro inflammation and functional network connectivity is relevant to cognitive impairment. We suggest that future studies should address how neuroinflammation relates to network function as $\mathrm{AD}$ progresses, and whether the neuroinflammation in $\mathrm{AD}$ is reversible, as the basis of immunotherapeutic strategies to slow the progression of AD.

\section{Introduction}

Neuroinflammation plays a key role in the etio-pathogenesis of Alzheimer's disease (AD) and other neurodegenerative disorders

Received Oct. 5, 2018; revised March 25, 2019; accepted April 11, 2019.

Author contributions: L.P., K.A.T., J.T.O., and J.B.R. designed research; L.P., W.R.B.-J., and R.A. performed research; L.P., K.A.T., P.S.J., and R.J.B. analyzed data; L.P., K.A.T., P.S.J., J.B.R., R.A., R.J.B., E.M., L.S., J.T.O., wrote the paper.
(Edison et al., 2008; Fernández-Botran et al., 2011; Fan et al., 2015; Stefanetti et al., 2016). Preclinical models (Heppner et al.,

This work was supported by the National Institute for Health Research Cambridge Biomedical Research Centre and Biomedical Research Unit in Dementia (NIHR; RG64473), the Wellcome Trust (103838 to J.B.R.), the Medical Research Council (RG91365/SUAG/004 and MR/P01271X/1), Alzheimer's Research UK to L.S (ARUK-SRF2017B-1), and by a British Academy Postdoctoral Fellowship to K.A.T. (PF160048). We thank our volunteers and the radiographers/technologists at WBIC and PET/CT, Addenbrooke's Hospital, for their invaluable support in data acquisition 
2015; Hoeijmakers et al., 2016; Villegas-Llerena et al., 2016; Li et al., 2018; Wang et al., 2018), and research in humans (FernándezBotran et al., 2011; Edison et al., 2013; Fan et al., 2015; Stefaniak and O'Brien, 2016; Passamonti et al., 2018), demonstrate that microglia, the brain's innate immune system, are activated in $\mathrm{AD}$ and other neurodegenerative diseases. Furthermore, genetic association studies have demonstrated a link between $\mathrm{AD}$ and polymorphisms or mutations in genes linked to immune response (Villegas-Llerena et al., 2016). Although the mechanisms and mediators of inflammatory risk in $\mathrm{AD}$ are not fully understood, synaptic and neuronal injury may arise from the release of cytokines and proinflammatory molecules such as interleukin- $1 \beta$ and TGF- $\beta$ (Fernández-Botran et al., 2011), or direct microglial injury to synapses (Hong and Stevens, 2016; Hong et al., 2016). These, in turn, impair synaptic function, network communication, and may accelerate neurodegeneration and synaptic loss (Heppner et al., 2015; Hoeijmakers et al., 2016; Villegas-Llerena et al., 2016; Li et al., 2018; Wang et al., 2018).

Clinical studies of neuroinflammation in dementia have exploited positron emission tomography (PET) ligands that bind to the mitochondrial translocator protein (TSPO) in activated microglia (Cagnin et al., 2001; Gerhard et al., 2006a,b; Edison et al., 2008, 2013; Fan et al., 2015; Passamonti et al., 2018). For example, relative to controls, patients with $\mathrm{AD}$ have higher $\left[{ }^{11} \mathrm{C}\right] \mathrm{PK} 11195$ binding in the hippocampus, other medial-temporal lobe regions, and posterior cortices such as the precuneus (Passamonti et al., 2018).

These findings raise the possibility of immunotherapeutic strategies to prevent or slow the progression of $\mathrm{AD}$. However, key issues remain to be resolved before such therapeutic strategies can be realized. For example, it is necessary to show how neuroinflammation is linked to cognitive deficits. A critical and unanswered question is whether regional neuroinflammation changes the functional connectivity of large-scale networks. Such largescale neural networks represent an intermediate phenotypic expression of pathology in many diseases that can be non-invasively quantified with resting-state functional magnetic resonance imaging (fMRI). A challenge is that neither the anatomical substrates of cognition nor the targets of neurodegenerative disease are mediated by single brain regions: they are in contrast distributed across multivariate and interactive networks.

We thus undertook a multimodal and multivariate neuroimaging study to combine $\left[{ }^{11} \mathrm{C}\right] \mathrm{PK} 11195$ quantification of distributed neuroinflammation with resting-state functional imaging in patients at different stages of AD. We used "source-based inflammetry" (SBI; analogous to "volumetry") to reduce the dimensionality (i.e., complexity) of the neuroinflammation signal, and used multiple

the NIHR Eastern Dementias and Neurodegenerative Diseases Research Network for help with subject recruitment; and Dr Istvan Boros and others at Wolfson Brain Imaging Centre RPU for the manufacture of $\left[{ }^{11} \mathrm{C}\right]$ PK11195 and $\left[{ }^{1} \mathrm{C}\right] \mathrm{PiB}$.

*L.P. and K.A.T. are co-first authors.

†J.T.O. and J.B.R. are co-last authors.

J.T.O. has served as deputy editor of International Psychogeriatrics, received Grant support from Avid (Lilly), and served as a consultant for Avid and GE Healthcare, all for matters not related to the current study. J.B.R. serves as editor to Brain, has been a consultant for Asceneuron and Syncona, and has received academic Grant funding from AZ-Medlmmune, Janssen, and Lilly, unrelated to this study. The remaining authors declare no competing financial interests.

Correspondence should be addressed to L. Passamonti at Ip337@medschl.cam.ac.uk.

https://doi.org/10.1523/JNEUROSCI.2574-18.2019

Copyright $@ 2019$ Passamonti et al.

This is an open-access article distributed under the terms of the Creative Commons Attribution License Creative Commons Attribution 4.0 International, which permits unrestricted use, distribution and reproduction in any medium provided that the original work is properly attributed.

$\begin{aligned} & \text { Table 1. Participant details (mean, with SD and range in parentheses) and group } \\
& \text { differences by } \boldsymbol{\chi}^{2} \text { test, one-way ANOVA, or independent samples } t \text { test }\end{aligned}$
\begin{tabular}{llll} 
Demographic and & $\mathrm{AD} / \mathrm{MCl}+$ & Controls & $\mathrm{AD} / \mathrm{MCl}+<$ \\
clinical data & $(N=28)$ & $(N=14)$ & Controls \\
\hline Sex, females/males & $12 / 16$ & $8 / 6$ & $\mathrm{NS}$ \\
Age, years (SD, range) & $72.7( \pm 8.5,53-86)$ & $68.3( \pm 5.4,59-81)$ & $\mathrm{NS}$ \\
Education, years & $12.9( \pm 3.0,10-19)$ & $14.1( \pm 2.7,10-19)$ & $\mathrm{NS}$ \\
$\begin{array}{l}\text { (SD, range) } \\
\text { MMSE (SD, range) }\end{array}$ & $25.6( \pm 2.2,21-30)$ & $28.8( \pm 1.0,27-30)$ & $\mathrm{t}=4.9, p<0.0001$ \\
ACE-R (SD, range) & $78.9( \pm 7.7,62-91)$ & $91.6( \pm 5.3,79-99)$ & $\mathrm{t}=5.5, p<0.0001$ \\
RAVLT (SD, range) & $1.5( \pm 1.6,0-6)$ & $9.6( \pm 3.2,3-15)$ & $\mathrm{t}=10.8, p<0.0001$ \\
\hline
\end{tabular}

NS, Not significant with $p>0.05$ (uncorrected).

linear-regression models to associate neuroinflammation, functional network connectivity, and cognition.

We tested two hypotheses: (1) that spatially distributed neuroinflammation related to significant changes in large-scale functional connectivity in patients with $\mathrm{AD}$, relative to controls. (2) That the relationship between neuroinflammation and abnormal functional connectivity mediates cognitive deficit in $\mathrm{AD}$.

\section{Materials and Methods}

\section{Participants}

The study was conducted in the context of the Neuroimaging of Inflammation in MemoRy and Other Disorders (NIMROD) study (BevanJones et al., 2017). We included 14 patients meeting clinical diagnostic criteria for probable $\mathrm{AD}$ (McKhann et al., 2011), and 14 patients with mild cognitive impairment (MCI); (12 females and 16 males in total) defined by: (1) a mini-mental score examination MMSE >24/30; (2) memory impairment at least 1.5 standard deviation (SD) below that expected for age and education (Petersen et al., 1999); and (3) biomarker evidence of amyloid pathology [positive Pittsburgh Compound-B PET scan (MCI+); Okello et al., 2009]. We combined patients with clinical $\mathrm{AD}$ and $\mathrm{MCI}+$ on the basis that these two groups represent a continuum of the same clinical spectrum (Okello et al., 2009).

Fourteen age-, sex-, and education-matched healthy controls (8 females, 6 males) with no history of major psychiatric or neurological illnesses, head injury, or any other significant medical comorbidity were also recruited. All participants were aged $>50$ years, with premorbid proficiency in English for cognitive testing, and had no acute infectious or chronic symptomatic systemic inflammatory disorder (e.g., lupus, rheumatoid arthritis, etc.), or contraindications to MRI. Patients were identified from the Cambridge University Hospitals NHS Trust Memory Clinics and the Dementias and Neurodegenerative Diseases Research Network (DeNDRoN), whereas healthy controls were recruited via DeNDRoN. All participants had mental capacity and gave written consent in accordance with the Declaration of Helsinki. The study was approved by the local research ethics committee.

\section{Clinical and cognitive assessment}

Clinical indices of cognitive deficit included Mini Mental State Examination (MMSE), Addenbrooke's Cognitive Examination-Revised (ACE$\mathrm{R})$, and Rey auditory verbal learning test (RAVLT). The demographic and neuropsychological measures are reported in Table 1. A principal component analysis (PCA) on the total MMSE, ACE-R, and RAVLT scores was conducted to reduce the dimensionality of the cognitive deficit into one latent variable, which summarized the largest portion of shared variance as the first principal component.

\section{Experimental design}

Structural and fMRI protocols and preprocessing. Structural and functional MRI were performed using a 3-tesla Siemens Tim Trio scanner with a 32-channel phased-array head coil. A T1-weighted magnetizationprepared rapid gradient-echo image was acquired with repetition time $=$ $2300 \mathrm{~ms}$, echo time $=2.98 \mathrm{~ms}$, matrix $=256 \times 240$, in-plane resolution of $1 \times 1 \mathrm{~mm}, 176$ slices of $1 \mathrm{~mm}$ thickness, inversion time $=900 \mathrm{~ms}$ and flip angle $=9$ degrees. The coregistered $\mathrm{T} 1$ images were used in a single- 
channel segmentation to extract probabilistic maps of six tissue classes: gray matter, white matter, cerebro-spinal fluid (CSF), bone, soft tissue, and background noise. The native-space gray-matter and white-matter images were submitted to diffeomorphic registration to create group template images (Ashburner, 2007). The template was normalized to the Montreal Neurological Institute (MNI) template using a 12-parameter affine transformation. After applying the normalization parameters from the T1 stream to warp preprocessed functional images into MNI space, the normalized images were smoothed using an $8 \mathrm{~mm}$ Gaussian kernel. An estimate of total gray matter, used in between-subject analysis as a covariate of no interest, was calculated as the median gray-matter tissue intensity in a group mask based on voxels with gray-matter tissue probability of 0.3 across all individuals. Resting-state multi-echo functional imaging was performed for $11 \mathrm{~min}$. A total of 269 echoplanar image volumes were acquired with repetition time $=2430 \mathrm{~ms}$, echo times $=$ $13.00,30.55$, and $48.10 \mathrm{~ms}$, matrix $=64 \times 64$, in-plane resolution of $3.75 \times 3.75 \mathrm{~mm}, 34$ slices of $3.8 \mathrm{~mm}$ thickness with an inter-slice gap of $0.38 \mathrm{~mm}$, and a generalized autocalibrating partial parallel acquisition (GRAPPA) imaging with an acceleration factor of 2 and bandwidth = $2368 \mathrm{~Hz} /$ pixel. The first six volumes were discarded to eliminate saturation effects and achieve steady-state magnetization. Pre-processing of resting-state data used the Multi-Echo Independent Components Analysis (ME-ICA) pipeline, which uses independent component analysis to classify blood oxygenation level-dependent (BOLD) and non-BOLD signals based on the identification of linearly dependent and independent echo-time related components (https://wiki.cam.ac.uk/bmuwiki/MEICA; Kundu et al., 2013). This provides an optimal approach to correct for movement-related and non-neuronal signals, and is therefore particularly suited to our study, in which systematic differences in head position might have been expected between groups. After ME-ICA, the data were smoothed with $5.9 \mathrm{~mm}$ full-width half-maximum Gaussian kernel.

The location of the key brain regions in each network was identified by spatial independent component analysis (ICA) using the Group ICA of fMRI Toolbox (Calhoun et al., 2001) in an independent dataset of 298 age-matched healthy individuals from the population-based cohort in the Cambridge Centre for Aging and Neuroscience (Cam-CAN; Shafto et al., 2014). Details about pre-processing and node definition are published previously (Tsvetanov et al., 2016). Four networks were identified by spatially matching to pre-existing templates (Shirer et al., 2012). The default mode network (DMN) contained five nodes: the ventral anterior cingulate cortex (vACC), dorsal, and ventral posterior conjugate cortex (PCC), and right and left inferior parietal lobules (IPL). The frontoparietal network (FPN) was defined using bilateral superior frontal gyrus (SFG) and angular gyrus (AG). Subcortical (SC) nodes included brain regions having differential group accumulation of $\left[{ }^{11} \mathrm{C}\right] \mathrm{PK} 11195$, namely, bilateral putamen and hippocampus. The node time-series were defined as the first principal component resulting from the singular value decomposition of voxels in an 8-mm-radius sphere, which was centered on the peak voxel per each node (Tsvetanov et al., 2016).

After extracting nodal time-series we sought to reduce the effects of noise confounds on functional connectivity effects of node time-series using a general linear model (Geerligs et al., 2017). This model included linear trends, expansions of realignment parameters, as well as average signal in the white-matter and CSF, including their derivative and quadratic regressors from the time-courses of each node (Satterthwaite et al., 2013). The signals in the white-matter and CSF were created by using the average across all voxels with corresponding tissue probability $>0.7$ in associated tissue probability maps available in the SPM12 software (https://www.fil.ion.ucl.ac.uk/spm/software/spm12/). A bandpass filter $(0.0078-0.1 \mathrm{~Hz})$ was implemented by including a discrete cosine transform set in the general linear model, ensuring that nuisance regression and filtering were performed simultaneously (Hallquist et al., 2013; Lindquist et al., 2019). The total head motion for each participant, which was used in subsequent between-subject analysis as a covariate of no interest (Geerligs et al., 2017), was quantified using the approach reported by Jenkinson et al. (2002), i.e., the root mean square of volumeto-volume displacement. Finally, the functional connectivity between each pair of nodes was computed using Pearson's correlation on postprocessed time-series.
PET protocols and preprocessing. All participants underwent $\left[{ }^{11} \mathrm{C}\right]$ PK11195 PET imaging to assess the extent and distribution of neuroinflammation while patients with MCI also underwent $\left[{ }^{11} \mathrm{C}\right] \mathrm{PiB}$ (Pittsburgh compound-B PET) scanning to evaluate the degree of $\beta$-amyloid accumulation. $\left[{ }^{11} \mathrm{C}\right] \mathrm{PK} 11195$ and $\left[{ }^{11} \mathrm{C}\right] \mathrm{PiB}$ PET were produced with high radiochemical purity $(>95 \%)$, with $\left[{ }^{11} \mathrm{C}\right] \mathrm{PiB}$ PET having a specific activity $>150 \mathrm{GBq} / \mu \mathrm{mol}$ at the end of synthesis, whereas $\left[{ }^{11} \mathrm{C}\right] \mathrm{PK} 11195$ specific activity was $\sim 85 \mathrm{GBq} / \mu \mathrm{mol}$ at the end of synthesis. PET scanning used a GE Advance PET scanner (GE Healthcare) and a GE Discovery $690 \mathrm{PET} / \mathrm{CT}$, with attenuation correction provided by a $15 \mathrm{~min} 68 \mathrm{Ge} /$ $68 \mathrm{Ga}$ transmission scan and a low dose computed tomography scan, respectively. The emission protocols were $550 \mathrm{MBq}$ [11C]PiB injection followed by imaging from 40 to $70 \mathrm{~min}$ postinjection, and $75 \mathrm{~min}$ of dynamic imaging ( 55 frames) starting concurrently with a $500 \mathrm{MBq}$ $\left[{ }^{11} \mathrm{C}\right]$ PK11195 injection. Each emission frame was reconstructed using the PROMIS 3-dimensional filtered back projection algorithm into a $128 \times 128$ matrix $30 \mathrm{~cm}$ trans-axial field-of-view, with a transaxial Hann filter cutoff at the Nyquist frequency (Kinahan and Rogers, 1989). Corrections were applied for randoms, dead time, normalization, scatter, attenuation, and sensitivity.

For $\left[{ }^{11} \mathrm{C}\right] \mathrm{PiB}$ we used reference tissue region-of-interest (ROI) defined by $\geq 90 \%$ on the SPM8 gray-matter probability map (smoothed to PET resolution) in the cerebellar cortex (Schuitemaker et al., 2007). For $\left[{ }^{11} \mathrm{C}\right] \mathrm{PK} 11195$, supervised cluster analysis was used to determine the reference tissue time-activity curve (Turkheimer et al., 2007). $\left[{ }^{11} \mathrm{C}\right] \mathrm{PiB}$ data were quantified using standardized uptake value ratio (SUVR) by dividing the mean CSF corrected radioactivity concentration in each Hammers atlas ROI by the corresponding mean CSF-corrected radioactivity concentration in the reference tissue ROI (whole cerebellum). $\left[{ }^{11} \mathrm{C}\right] \mathrm{PiB}$ data were treated as dichotomous measures (i.e., positive or negative) and considered positive if the average SUVR value across the cortical ROIs was $>1.5$ (Hatashita and Yamasaki, 2010). For $\left[{ }^{11} \mathrm{C}\right]$ PK11195 maps of non-displaceable binding potential $\left(\mathrm{BP}_{\mathrm{ND}}\right)$, a measure of specific binding, were determined using a basis function implementation of the simplified reference tissue model, both with and without CSF contamination correction (Gunn et al., 1997). $\left[{ }^{11} \mathrm{C}\right] \mathrm{PK} 11195 \mathrm{BP}_{\mathrm{ND}}$ maps (termed from now on PK maps for simplicity) were also generated using this basis function approach.

The PK maps were coregistered and warped to the MNI space using the flow fields. To minimize the noise effects from non-gray-matter regions, the normalized PK maps were masked with a group-based gray-matter mask based on voxels having gray-matter tissue probability larger than 0.3 in gray-matter segmented images across all individuals. The normalized images were smoothed using a $6 \mathrm{~mm}$ Gaussian kernel. We then used independent component analysis across participants to derive spatial patterns of PK maps across voxels expressed by the group in a small number of independent components. All PK maps were spatially concatenated and submitted to Source Based Inflammetry (SBI) to decompose images across all individuals in a set of spatially independent sources without providing any information about the group (Xu et al., 2009), using the GIFT toolbox. Specifically, the $n$-by- $m$ matrix of participantsby-voxels was decomposed into: (1) a source matrix that maps each independent component to voxels (here referred to as $\mathrm{PK}_{\mathrm{IC}}$ maps), and (2) a mixing matrix that maps $\mathrm{PK}_{\mathrm{ICs}}$ to participants. The mixing matrix consists of loading values (1 per participant) indicating the degree to which a participant expresses a defined $\mathrm{PK}_{\mathrm{IC}}$. The independent component loading values for the $\mathrm{PK}_{\mathrm{IC}}$ were taken forward to betweenparticipant analysis of functional connectivity (Fig. 1), if they were (1) differentially expressed by controls vs. patients with $\mathrm{AD}$ pathology; and (2) were associated with atrophy (see Results and Fig. 3). Only one dependent variable (IC3) met these criteria.

\section{Statistical analyses}

We adopted a two-level procedure, in which, at the first-level, we sought to identify functional connectivity differences associated with differences in $\left[{ }^{11} \mathrm{C}\right] \mathrm{PK} 11195$ binding. In a second-level analysis, we tested whether individual variability in functional connectivity (from first-level analysis) is specifically associated with variability in cognitive deficit in the group of patients with AD pathology. 


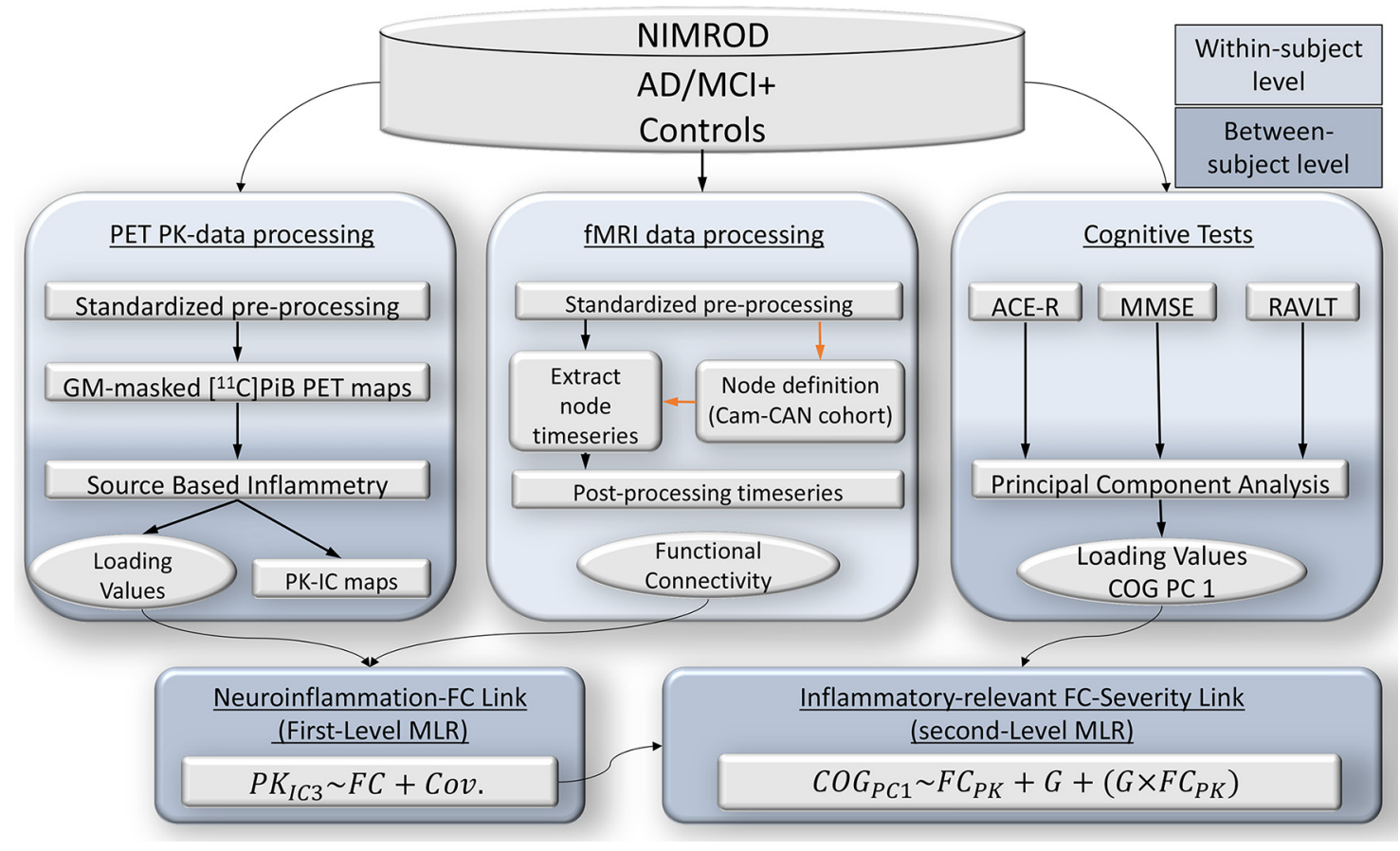

Figure 1. Schematic representation of various modality datasets in the study, their processing pipelines on a within-subject level (light blue), as well as data-reduction techniques and statistical strategy on between-subject level (dark blue) to test for associations between the datasets. FC, Functional connectivity; Cov, covariates; COG PC1, latent variable (cognitive deficit, which summarizes the largest portion of shared variance as the first principal component); GM, gray matter. NIMROD study (Neuroimaging of Inflammation in Memory and Other Disorders), AD/MCI + , Alzheimer's Disease/Mild Cognitive Impairment (positive PET amyloid, 11C PiB PET), PET PK, positron emission tomography [11C]PK11195 ligand (microglia activation), PK-IC maps, PET [11C]PK11195 independent component maps, PK IC3, 3rd component of [11C]PK11195 ligand maps, 11CPiB PET (amyloid PET), fMRI, functional magnetic resonance imaging, Cam-CAN, Cambridge Centre for Ageing and Neuroscience, G, diagnostic group (AD/MCI + or Controls), ACE-R, Addenbrookes' Cognitive Examination - revised test, MMSE, mini-mental status examination, RAVLT, Ray Audio-Visual Learning Test, MLR, multiple linear regression.

Details about the first-level analysis approach are published previously (Tsvetanov et al., 2018). In short, we used multiple linear regression (MLR) with well conditioned shrinkage regularization (Ledoit and Wolf, 2004) to identify correlated structured sources of variance between functional connectivity and neuroinflammation measures. In particular, this analysis describes the linear relationship between functional connectivity and PK maps on a between-subject level, in terms of structure coefficients (Thompson and Borrello, 1985), by providing a linear combination of the functional connectivity measures, which we term brain scores, that are optimized to be highly correlated with the between-subject variability in the expression of the PK maps. Namely, brain-wide connectivity strength for each individual defined the independent variables, and $\mathrm{PK}_{\mathrm{IC}}$ subject-specific loading values for group differentiating components were used as a dependent variable.

To identify and exclude potential outliers, Grubbs' test was used (Grubbs, 1969; Barnett and Lewis, 1994). None of the loading values in the IC3 was outlying observation. Furthermore, to down-weight the effects of extreme or imprecise data points, the analyses used robust linear regression.

To avoid overfitting, first-level multiple linear regression model was integrated with a fivefold cross-validation approach (Thompson and Borrello, 1985). To minimize the non-negligible variance of traditional $k$-fold cross-validation procedure, we repeated each $k$-fold 1,000 times with random partitioning of the folds to produce an $R$ value distribution, for which we report the median value.

Next, we tested the hypothesis that the effect of neuroinflammation on functional connectivity was related to cognitive deficit, in patients relative to controls. To this end, we performed a second-level multiple linear regression (MLR) analysis. Independent variables included subjects' brain scores from first-level MLR (reflecting how strongly each individual expressed the whole-brain pattern of functional connections weighted by the IC3-PET-derived data), group information, and their interaction term (brain scores $\times$ group).
In other words, we examined the linear relationship between functional connectivity and expressions of PK maps on a between-subject level, in terms of structure coefficients (Thompson and Borrello, 1985). Hence, we used multiple linear regression to identify a linear combination of the functional connectivity measures, which we term brain scores, that were optimized to be highly correlated with the between-subject variability in the expression of PK maps. More specifically, the wholebrain connectivity strength for each individual defined the independent variables $(m \times n)$, and $\mathrm{PK}_{\mathrm{IC}}$ subject-specific loading values for the group differentiating component (IC3) were used as dependent variable $(m \times$ 1 ), where $m$ is the number of subjects and $n$ is the number of pairwise connections. This produced a set of structure coefficients $(1 \times n)$ and a set of subject scores $(m \times 1)$ for the functional connectivity data. The structure coefficients reflect the contribution of each connection to the overall functional connectivity pattern; conceptually similar to the component loadings in a PCA on cognitive data and the source matrix in group ICA on PET-PK data (here called SBI). The brain scores indicated how strongly an individual expresses the functional connectivity pattern, which is conceptually similar to the subject-specific scores in a PCA and subject-specific loadings in the SBI analysis. These brain scores were correlated with the subjects' cognitive performance, in a second-level multiple linear-regression analysis. For the cognitive performance, we did not choose a single test, but rather a summarized cognitive ability score in terms of the first principal component across three cognitive tests.

Covariates of no interest included age, sex, head movement, and global gray-matter volume.

\section{Results}

Source-based inflammetry (SBI)

The optimal number of components $(n=5)$ was detected with minimum-distance length criteria. One component showed significant differences between the patient and control group in 

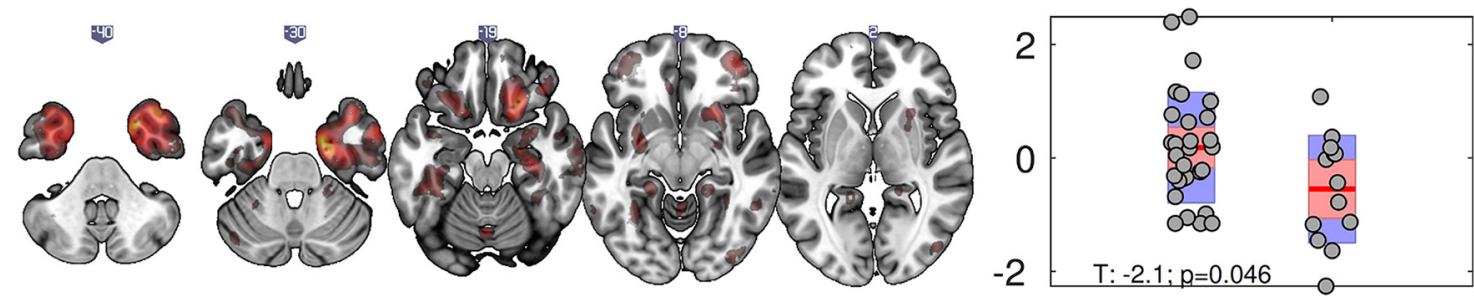

\section{$\mathrm{AD} / \mathrm{MCl}+\mathrm{HC}$}

Figure 2. Source Based Inflammetry (SBI) for the component differentially expressed between groups: (left) independent component spatial map reflecting increase in PK ([11C]PK11195 ligandactivated microgia) binding values in cortical and subcortical areas (red blobs) including inferior temporal cortex and hippocampus, these regionally specific increases are over and above the global PK differences between groups. Right, Bar plot of subject loading values for $\mathrm{AD} / \mathrm{MCl}+$ and control group (each circle represents an individual) indicating higher loading values for $\mathrm{AD} / \mathrm{MCl}+$ than control group as informed by two-sample unpaired permutation test (a robust linear regression was used to down-weight the effects of extreme data points). AD/MCI+, Alzheimer's Disease/Mild Cognitive Impairment (positive PET amyloid, 11C PiB PET), HC, healthy controls.
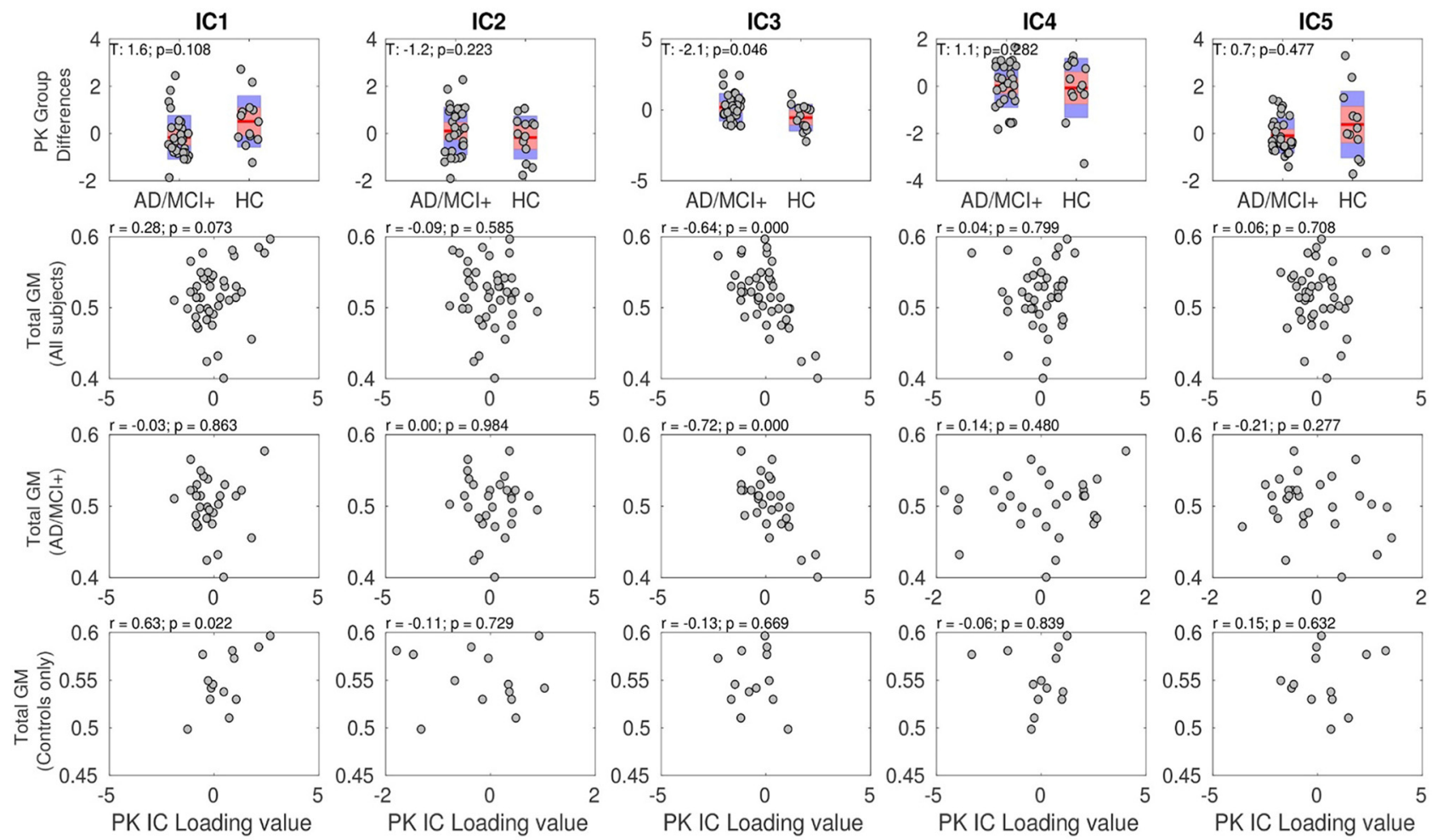

Figure 3. The Source Based Inflammetry (SBI) identified five independent components (ICS) that reflected PK ([11C]PK11195 ligand-activated microgia) binding values in cortical and subcortical areas. The PKIC3 component differed between AD/MCI + patients and controls (first row, third column). This PKIC3 component negatively correlated with total gray-matter volumes in all individuals as well as in patients only (but not controls only; third column and second, third, and fourth rows). In other words, the patients expressing higher $\left.{ }^{11} \mathrm{C}\right]$ PK11195 binding PKIC3 component (reflecting higher binding in the inferior temporal cortex and hippocampus as shown in Fig. 2) displayed higher levels of brain-wide atrophy. GM, gray matter, AD/MCI+, Alzheimer's Disease/Mild Cognitive Impairment (positive PET amyloid, 11C PiB PET), HC, healthy controlls.

terms of their loading values $\left(\mathrm{PK}_{\mathrm{IC} 3}, t=-2.1, p=0.04\right.$; Fig. 2 right, robust linear regression). The spatial extent of this $\mathrm{PK}_{\mathrm{IC} 3}$ included voxels with high values in cortical and subcortical regions, including the inferior temporal cortex and hippocampus, indicating that individuals with higher loading values, in this case the patient group, had higher $\left[{ }^{11} \mathrm{C}\right] \mathrm{PK} 11195$ binding in these regions, relative to the control group (Fig. 2, left). The other components did not differentiate patients from controls (Fig. 3, first row).

The $\mathrm{PK}_{\mathrm{IC}}$ component, which differed between patients and controls, was also the sole PK component that negatively correlated with total gray-matter values in patients but not controls (Fig. 3, third column, and second and third rows). In other words, the patients expressing higher $\left[{ }^{11} \mathrm{C}\right] \mathrm{PK} 11195$ binding showed also higher levels of cortical atrophy (Fig. 3, second and third rows). This result was obtained when including the graymatter volume as a covariate of no interest in the analysis, which suggests that the reported association was over and above the effects of overall brain atrophy.

All in all, our findings imply that the $\mathrm{PK}_{\mathrm{IC} 3}$ component reflects specific patterns of neuroinflammation and neurodegeneration in $\mathrm{AD}$. These patterns were next tested in terms of their relevance for changes in large-scale network function and their interactive effect in mediating cognitive deficit in $\mathrm{AD}$.

\section{Functional connectivity}

As expected, there was strong positive functional connectivity between all nodes within the four networks, identified by spatially 


\section{Mean Effects}

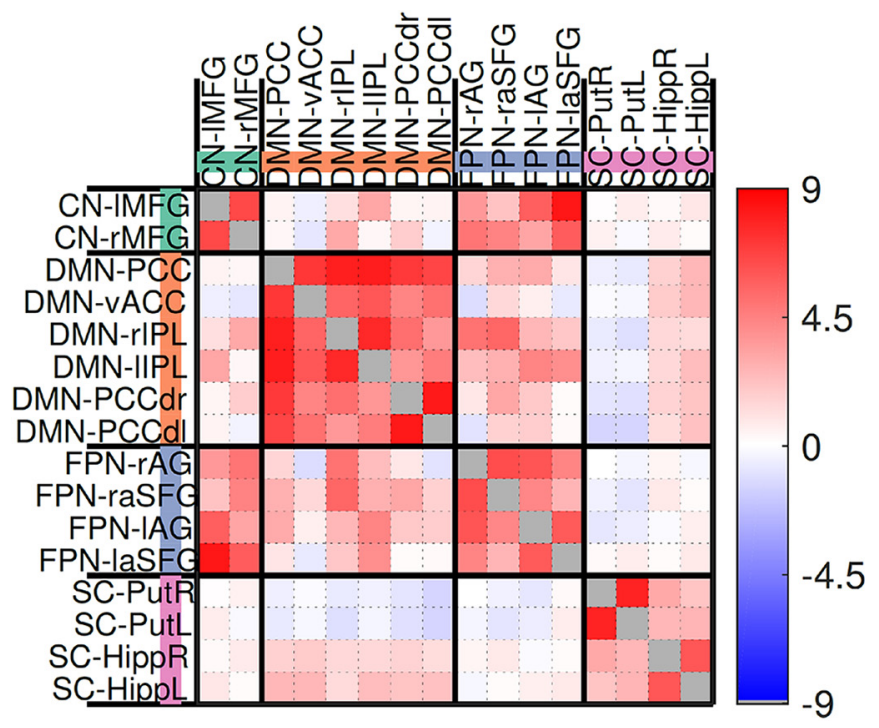

\section{$\mathrm{AD} / \mathrm{MCl}+>$ Controls}

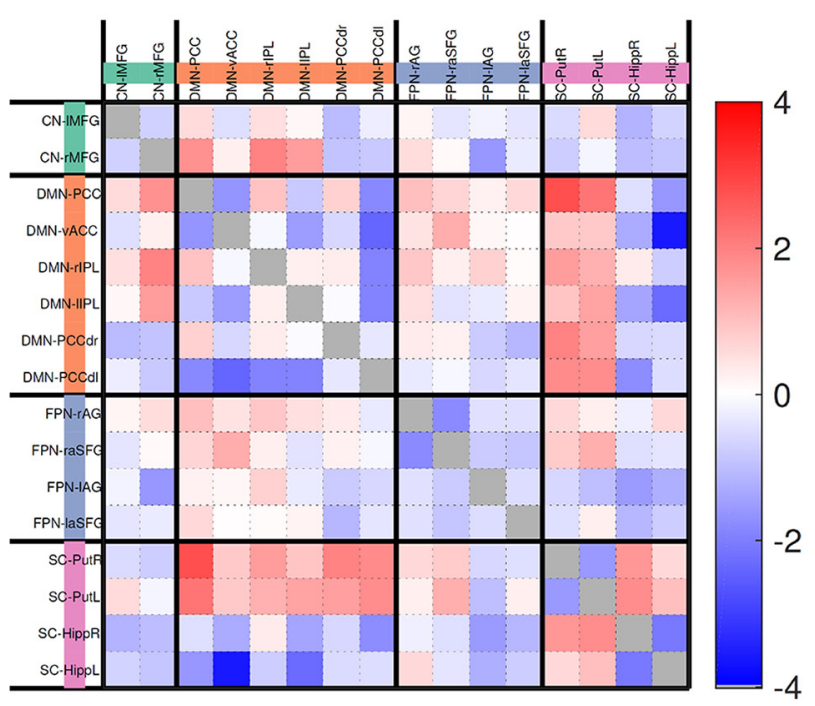

Figure 4. Mean effects (left) and group difference effects ( $\mathrm{AD} / \mathrm{MCI}+>$ Controls, right) between default mode network (DMN) and subcortical (SC) regions using univariate approach. vACC, ventral Anterior cingulate cortex; PCC, posterior cingulate cortex; IPL, intraparietal lobule; FPN, frontoparietal network; Put, putamen; Hipp, hippocampus, AG, angular gyrus; CN-MFG, middle frontal gyrus (cortical network), SFG, superior frontal gyrus; r, right; I, left. Note that the whole pattern of brain connectivity rather than each connection separately was used to study how subject-specific neuroinflammatory levels influence large-scale network connectivity (Fig. 5). AD/MCI+, Alzheimer's Disease/Mild Cognitive Impairment (positive PET amyloid, 11C PiB PET).

matching to pre-existing templates (Fig. 4, left). In terms of group differences, the functional connectivity within networks (within the default mode network, within the frontoparietal network, left-right putamen and left-right hippocampus) and between the default mode network and hippocampus was weaker in patients relative to controls (Fig. 4, right). Furthermore, the connectivity between the putamen and hippocampus increased, whereas the connectivity between default mode network and putamen was less negative for patients relative to controls.

\section{Functional connectivity and neuroinflammation}

The first-level multiple linear-regression model assessing the relationship between $\mathrm{PK}_{\mathrm{IC} 3}$ maps and functional connectivity data was significant $(r=0.52, p<0.001)$. The standard coefficients indicated positive and negative associations between the $\mathrm{PK}_{\mathrm{IC} 3}$ loading values and between-subjects variability in functional connectivity (Fig. 5, left). In other words, individuals with higher $\left[{ }^{11} \mathrm{C}\right] \mathrm{PK} 11195$ binding values in the inferior temporal cortex and medial temporal lobe regions (as reflected by higher $\mathrm{PK}_{\mathrm{IC} 3}$ values) showed: (1) increased connectivity between the default mode network, the hippocampus, and other subcortical regions; and (2) weaker connectivity for nodes within the default mode network.

\section{Linking neuroinflammation, connectivity, and cognitive deficits}

The first component of the PCA of cognitive tests explained $80 \%$ of the variance across the three cognitive measures (with coefficients of $0.61,0.61$, and 0.52 for MMSE, ACE-R, and RAVLT, respectively).

Next, we tested whether the effects of neuroinflammation on network connectivity were specific to the patient group and whether this functionally-relevant neuroinflammation related to cognitive deficits. Consistently with this hypothesis, the interaction term between group and brain scores (reflecting how strongly each individual expresses the pattern shown in Fig. 5 left, which is the brain-wide pattern of functional connections optimized to highly correlate with the IC3-PET-derived data) was significantly associated to the first component of the PCA of cognitive tests $(t=-3.4, p=0.004)$.

A post hoc analysis within each group indicated a significant negative association between the behavioral scores from the PCA and functional connectivity/PK-combined indices in the patient group ( $r=-0.51, p=0.005$; Fig. 5 , right). Conversely, a nonsignificant positive direction of association for the same relationship between PCA-derived cognitive scores and brain measures was found in controls $(r=0.46, p=0.09)$. The significant difference between patients and controls remains if $\mathrm{AD}$ and MCI+ subgroups are analyzed separately (data not shown). The negative association in the patient group indicated that patients in whom higher neuroinflammation was more strongly associated with more abnormal connectivity also performed worse on a summary measure of cognitive deficit.

\section{Discussion}

This study establishes a link between the presence of neuroinflammation and the disruption of large-scale functional connectivity in $\mathrm{AD}$. The degree to which patients expressed the association between abnormal functional connectivity and neuroinflammation itself correlated with their cognitive deficit. In other words, the patients' cognitive scores were correlated to a pattern of brain connectivity that was itself linked to microglia activation. This relationship between cognition and a PET-rs-fMRI association (i.e., neuroinflammation relevant functional connectivity) was only seen in patients with $\mathrm{AD}$, but not healthy controls. This suggests that not only does neuroinflammation relate to large-scale network function, but also that the disruption of connectivity linked to neuroinflammation mediates cognitive deficits in $\mathrm{AD}$.

We propose that the cognitive deficits in $\mathrm{AD}$ can be directly related to changes in functional connectivity which in turn are mediated by microglia activation, although we acknowledge that there are several mechanisms by which neuroinflammation can 


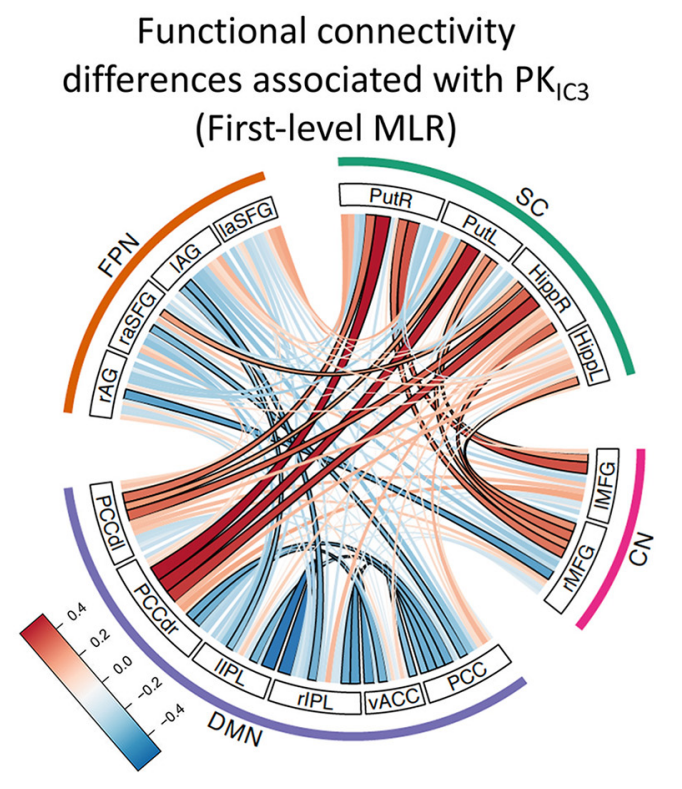

\section{Neuroinflammatory effects on functional connectivity with disease severity (Second-level MLR)}

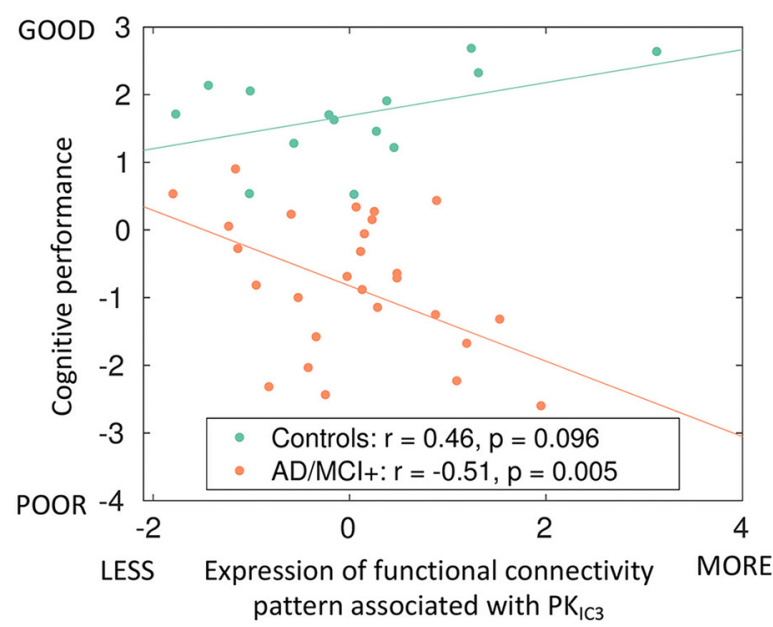

Figure 5. Left, First-level Multiple Linear Regression (MLR) indicating that functional connectivity differences (deviating from groups effects in Fig. 3) are associated positively with PK (PK PET ligand 3rd Independent Component). Connections surviving a threshold of $p<0.05$ corrected for multiple comparisons are highlighted with a black contour, although it is important to bear in mind that the whole-pattern of brain connectivity was used in the analysis shown on the right. Right, Second-level MLR association between PK $\mathrm{I}_{\mathrm{IC}}$ pattern of functional connectivity and cognitive performance for patients with AD pathology (including $\mathrm{MCl}+$; orange) and control (green) groups. The group difference in slopes was significant $(p<0.0001)$. By using multiple linear regression and correlations with cognitive performance, we found that the change in patients' cognition was correlated to a pattern of brain connectivity that was itself linked to neuro-inflammation. This relationship between cognition and a PET-rsfMRI association (i.e., neuroinflammation-functional connectivity) was only seen in AD/MCI + patients, not controls. vACC, ventral Anterior cingulate cortex; PCC, posterior cingulate cortex; IPL intraparietal lobule; FPN, frontoparietal network; SC, subcortical, DMN, default mode network, DMNd, dorsal DMN, Put, putamen; Hipp, hippocampus, AG, angular gyrus; $S F G$, superior frontal gyrus; $R$, right; $L$, left.

alter brain functional connectivity and vice versa (i.e., the ways in which synaptic firing can influence microglia). Microglia are important contributors in the process of synaptic pruning and regulation of synaptic function (Hong et al., 2016). The microglia's highly mobile and ramified branches can reach and surround synaptic terminals to promote phagocytosis and synaptic demise (Hong et al., 2016). Microglia-induced complement activation might also contribute to synaptic dysfunction and loss, especially in the context of amyloid deposition and neuritic plaque formation (Hong et al., 2016). On the other hand, synaptic firing can influence microglia activation via specific membrane receptors and ion channels (Tofaris and Buckley, 2018).

The anatomical distribution of neuroinflammation in $\mathrm{AD}$ and its effects on large-scale network function supports the hypothesis that neuroinflammation might be an early event in the pathogenesis of $\mathrm{AD}$ and that our current results are not driven by a global effect of a systemic inflammatory confound which would have affected the whole-brain indistinctively.

Our study has three implications. First, it supports the use of integrative or multimodal neuroimaging as a useful tool to improve our understanding of the brain determinants that mediate healthy and pathological aging (Geerligs and Tsvetanov, 2017).

Second, it reinforces the notion that neuroinflammation is a key pathophysiological mediator of $\mathrm{AD}$ and its clinical variability (Weiler et al., 2016). Genome-wide association studies have challenged the idea that neuroinflammation is merely a secondary event caused by neurodegeneration and have conversely sustained a primary role of microglia-related molecular pathways in the etio-pathogenesis of AD (Guerreiro et al., 2013; Jonsson et al., 2013). For instance, mutations in TREM 2 , an immune cells receptor expressed on microglia, represent a risk factor for $\mathrm{AD}$ and other neurodegenerative diseases (Guerreiro et al., 2013; Jonsson et al., 2013). Together with our results, these data suggest that immunotherapeutic strategies might be helpful to reduce the deleterious impact of neuroinflammation on cognitive deficits in $\mathrm{AD}$.

Third, the functional connectivity abnormalities observed here can be considered an intermediate phenotypic expression of the neuroinflammatory pathology in $\mathrm{AD}$. This can be relevant to reconcile the apparent conflict between the encouraging findings from basic research on the role of neuroinflammation in $\mathrm{AD}$ pathogenesis (Heppner et al., 2015) and the results from human studies, which as yet have provided little support for immunotherapeutics in AD (Adapt Research Group et al., 2007, 2008), despite epidemiological evidence (Breitner and Zandi, 2001; in't Veld et al., 2001).

In other words, assessing how neuroinflammation influences the intermediate phenotypes of large-scale network functional connectivity might help explaining why clinical trials have failed so far to demonstrate a role for immunotherapeutic strategies because of high patient heterogeneity. We showed marked individual differences in the relationship between resting-state functional connectivity and neuroinflammation in patients with $\mathrm{AD}$ at different stages, and it was this variance that was significantly related to individual differences in cognitive performance.

Our study has also limitations and caveats. First, we recognize that even the multivariate methods of statistical associations used here do not in themselves demonstrate causality between neuroinflammation, network dysfunction, and cognition. To address this issue, longitudinal and interventional studies are needed, alongside mediation analyses (Fan et al., 2015; Kreisl et al., 2016).

Second, the molecular pathology of $\mathrm{AD}$ is multifaceted, with amyloid deposition, tau accumulation, and vasculopathy. These processes, alone or in combination, may moderate the associa- 
tion between neuroinflammation and functional connectivity; hence, multimodal studies that capture each of these aspects will be useful to formally assess the complex interplay between neuroinflammation, abnormal tau deposition, vasculopathy, and cognitive deficits.

Third, the confounding effect of head motion on functional imaging has been fully recognized as both challenging and critical for interpretation of functional imaging studies, especially in clinical populations. To minimize such confound, we used ME-ICA and validated pre-processing pipelines, which separate changes in the fMRI signal that distinguish between BOLD and non-BOLD signals. Furthermore, we included movementrelated parameters as covariates of no interest in second-level analyses, as well as motion and physiological signals in first-level analyses.

Fourth, the use of the $\left[{ }^{11} \mathrm{C}\right] \mathrm{PK} 11195$ tracer has its own limitations in terms of reduced affinity to the mitochondrial TSPO in activated microglia, especially compared with second-generation TSPO tracers as PBR28 (Fujita et al., 2017). On the other hand, such second-generation TSPO tracers are affected by common genetic polymorphisms (Owen et al., 2012).

Fifth, at the phenotypic level, it remains to be determined whether the deleterious impact of neuroinflammation on network function can be revealed in pre-symptomatic individuals at risk of $\mathrm{AD}$, for example, in carriers of autosomal dominant genetic mutations. Despite the inclusion of patients with mild cognitive impairment with biomarker evidence of AD pathology, our study cannot resolve the timing of neuroinflammation and its causal relationship to network dysfunction, cell loss, and cognitive deficit. There is initial evidence that neuroinflammation may precede abnormal protein aggregation and brain atrophy in presymptomatic carriers of MAPT mutations (Bevan-Jones et al., 2019), although further studies are needed to confirm these preliminary findings in $\mathrm{AD}$ or other neurodegenerative diseases.

In conclusion, we have shown that SBI of $\left[{ }^{11} \mathrm{C}\right] \mathrm{PK} 11195$ PET data revealed a distributed profile of neuroinflammation in $\mathrm{AD}$, which in turn related to abnormal functional connectivity. Our crossmodal multivariate analyses also indicated that heterogeneity in cognitive status was associated to variability in neuroinflammation-related network dysfunction. These data emphasize the value of multi-modal neuroimaging to study how different aspects of the molecular pathology of AD mediate brain function and cognition. Improved stratification procedures may facilitate more efficient therapeutic trials in $\mathrm{AD}$, based not only on neuro inflammation, tau, atrophy, or connectivity, but on their complex interaction that leads to individual differences in cognitive impairment.

\section{References}

Adapt Research Group; Lyketsos CG, Breitner JC, Green RC, Martin BK, Meinert C, Piantadosi S, Sabbagh M (2007) Naproxen and celecoxib do not prevent $\mathrm{AD}$ in early results from a randomized controlled trial. Neurology 68:1800-1808.

Adapt Research Group; Martin BK, Szekely C, Brandt J, Piantadosi S, Breitner JC, Craft S, Evans D, Green R, Mullan M (2008) Cognitive function over time in the Alzheimer's disease anti-inflammatory prevention trial (ADAPT): results of a randomized, controlled trial of naproxen and celecoxib. Arch Neurol 65:896-905.

Ashburner J (2007) A fast diffeomorphic image registration algorithm. Neuroimage 38:95-113.

Barnett V, Lewis T (1994) Outliers in statistical data. Chichester: Wiley.

Bevan-Jones WR, Surendranathan A, Passamonti L, Vázquez Rodriguez P, Arnold R, Mak E, Su L, Coles JP, Fryer TD, Hong YT, Williams G, Aigbirhio F, Rowe JB, O'Brien JT (2017) Neuroimaging of inflammation in memory and related other disorders (NIMROD) study protocol: a deep phenotyping cohort study of the role of brain inflammation in dementia, depression and other neurological illnesses. BMJ Open 7:e013187.

Bevan-Jones WR, Cope TE, Jones PS, Passamonti L, Hong YT, Fryer T, Arnold R, Coles JP, Aigbirhio FI, O’Brien JT, Rowe JB (2019) In vivo evidence for pre-symptomatic neuroinflammation in a MAPT mutation carrier. Ann Clin Transl Neurol 6:373-378.

Breitner JC, Zandi PP (2001) Do nonsteroidal antiinflammatory drugs reduce the risk of Alzheimer's disease? N Engl J Med 345:1567-1568.

Cagnin A, Brooks DJ, Kennedy AM, Gunn RN, Myers R, Turkheimer FE, Jones T, Banati RB (2001) In-vivo measurement of activated microglia in dementia. Lancet 358:461-467.

Calhoun VD, Adali T, Pearlson GD, Pekar J (2001) A method for making group inferences from functional MRI data using independent component analysis. Hum Brain Mapp 14:140-151.

Edison P, Archer HA, Gerhard A, Hinz R, Pavese N, Turkheimer FE, Hammers A, Tai YF, Fox N, Kennedy A, Rossor M, Brooks DJ (2008) Microglia,amyloid,andcognitioninAlzheimer'sdisease:an $\left[{ }^{11} \mathrm{C}\right](\mathrm{R}) \mathrm{PK} 11195-$ PET and $\left[{ }^{11} \mathrm{C}\right]$ PIB-PET study. Neurobiol Dis 32:412-419.

Edison P, Ahmed I, Fan Z, Hinz R, Gelosa G, Ray Chaudhuri K, Walker Z, Turkheimer FE, Brooks DJ (2013) Microglia, amyloid, and glucose metabolism in Parkinson's disease with and without dementia. Neuropsychopharmacology 38:938-949.

Fan Z, Aman Y, Ahmed I, Chetelat G, Landeau B, Ray Chaudhuri K, Brooks DJ, Edison P (2015) Influence of microglial activation on neuronal function in Alzheimer's and Parkinson's disease dementia. Alzheimers Dement 11:608-621.e7.

Fernández-Botran R, Ahmed Z, Crespo FA, Gatenbee C, Gonzalez J, Dickson DW, Litvan I (2011) Cytokine expression and microglial activation in progressive supranuclear palsy. Parkinsonism Relat Disord 17:683-688.

Fujita M, Kobayashi M, Ikawa M, Gunn RN, Rabiner EA, Owen DR, Zoghbi SS, Haskali MB, Telu S, Pike VW, Innis RB (2017) Comparison of four ${ }^{11} \mathrm{C}$-labeled PET ligands to quantify translocator protein $18 \mathrm{kDa}$ (TSPO) in human brain: (R)-PK11195, PBR28, DPA-713, and ER176-based on recent publications that measured specific-to-non-displaceable ratios. EJNMMI Res 7:84

Geerligs L, Tsvetanov KA (2017) The use of resting state data in an integrative approach to studying neurocognitive ageing: commentary on Campbell and Schacter (2016). Lang Cogn Neurosci 32:684-691.

Geerligs L, Tsvetanov KA, Cam-Can, Henson RN (2017) Challenges in measuring individual differences in functional connectivity using fMRI: the case of healthy aging. Hum Brain Mapp 38:4125-4156.

Gerhard A, Pavese N, Hotton G, Turkheimer F, Es M, Hammers A, Eggert K, Oertel W, Banati RB, Brooks DJ (2006a) In vivo imaging of microglial activation with $\left[{ }^{11} \mathrm{C}\right](\mathrm{R})-\mathrm{PK} 11195 \mathrm{PET}$ in idiopathic Parkinson's disease. Neurobiol Dis 21:404-412.

Gerhard A, Trender-Gerhard I, Turkheimer F, Quinn NP, Bhatia KP, Brooks DJ (2006b) In vivo imaging of microglial activation with $\left[{ }^{11} \mathrm{C}\right](\mathrm{R})-$ PK11195 PET in progressive supranuclear palsy. Mov Disord 21:89-93.

Grubbs FE (1969) Procedures for detecting outlying observations in samples. Technometrics 11:1-21.

Guerreiro R, Wojtas A, Bras J, Carrasquillo M, Rogaeva E, Majounie E, Cruchaga C, Sassi C, Kauwe JS, Younkin S, Hazrati L, Collinge J, Pocock J, Lashley T, Williams J, Lambert JC, Amouyel P, Goate A, Rademakers R, Morgan K, et al. (2013) TREM2 variants in Alzheimer's disease. N Engl J Med 368:117-127.

Gunn RN, Lammertsma AA, Hume SP, Cunningham VJ (1997) Parametric imaging of ligand-receptor binding in PET using a simplified reference region model. Neuroimage 6:279-287.

Hallquist MN, Hwang K, Luna B (2013) The nuisance of nuisance regression: spectral misspecification in a common approach to resting-state fMRI preprocessing reintroduces noise and obscures functional connectivity. Neuroimage 82:208-225.

Hatashita S, Yamasaki H (2010) Clinically different stages of Alzheimer's disease associated by amyloid deposition with $\left[{ }^{11} \mathrm{C}\right]$-PIB PET imaging. J Alzheimers Dis 21:995-1003.

Heppner FL, Ransohoff RM, Becher B (2015) Immune attack: the role of inflammation in Alzheimer disease. Nat Rev Neurosci 16:358-372.

Hoeijmakers L, Heinen Y, van Dam AM, Lucassen PJ, Korosi A (2016) Microglial priming and Alzheimer's disease: a possible role for (early) immune challenges and epigenetics? Front Hum Neurosci 10:398.

Hong S, Stevens B (2016) Microglia: phagocytosing to clear, Sculpt, and Eliminate. Dev Cell 38:126-128. 
Hong S, Beja-Glasser VF, Nfonoyim BM, Frouin A, Li S, Ramakrishnan S, Merry KM, Shi Q, Rosenthal A, Barres BA, Lemere CA, Selkoe DJ, Stevens B (2016) Complement and microglia mediate early synapse loss in Alzheimer mouse models. Science 352:712-716.

in't Veld BA, Ruitenberg A, Hofman A, Launer LJ, van Duijn CM, Stijnen T, Breteler MM, Stricker BH (2001) Nonsteroidal antiinflammatory drugs and the risk of Alzheimer's disease. N Engl J Med 345:1515-1521.

Jenkinson M, Bannister P, Brady M, Smith S (2002) Improved optimization for the robust and accurate linear registration and motion correction of brain images. Neuroimage 17:825-841.

Jonsson T, Stefansson H, Steinberg S, Jonsdottir I, Jonsson PV, Snaedal J, Bjornsson S, Huttenlocher J, Levey AI, Lah JJ, Rujescu D, Hampel H, Giegling I, Andreassen OA, Engedal K, Ulstein I, Djurovic S, IbrahimVerbaas C, Hofman A, Ikram MA, et al. (2013) Variant of TREM2 associated with the risk of Alzheimer's disease. N Engl J Med 368:107-116.

Kinahan PE, Rogers JG (1989) Analytic 3D image reconstruction using all detected events. IEEE Trans Nucl Sci 36:964-968.

Kreisl WC, Lyoo CH, Liow JS, Wei M, Snow J, Page E, Jenko KJ, Morse CL, Zoghbi SS, Pike VW, Turner RS, Innis RB (2016) ${ }^{11} \mathrm{C}-\mathrm{PBR} 28$ binding to translocator protein increases with progression of Alzheimer's disease. Neurobiol Aging 44:53-61.

Kundu P, Brenowitz ND, Voon V, Worbe Y, Vértes PE, Inati SJ, Saad ZS, Bandettini PA, Bullmore ET (2013) Integrated strategy for improving functional connectivity mapping using multiecho fMRI. Proc Natl Acad Sci U S A 110:16187-16192.

Ledoit O, Wolf M (2004) A well-conditioned estimator for largedimensional covariance matrices. J Multivar Anal 88:365-411.

Li JW, Zong Y, Cao XP, Tan L, Tan L (2018) Microglial priming in Alzheimer's disease. Ann Transl Med 6:176.

Lindquist MA, Geuter S, Wager TD, Caffo BS (2019) Modular preprocessing pipelines can reintroduce artifacts into fMRI data. Hum Brain Mapp 40:2358-2376.

McKhann GM, Knopman DS, Chertkow H, Hyman BT, Jack CR Jr, Kawas CH, Klunk WE, Koroshetz WJ, Manly JJ, Mayeux R, Mohs RC, Morris JC, Rossor MN, Scheltens P, Carrillo MC, Thies B, Weintraub S, Phelps CH (2011) The diagnosis of dementia due to Alzheimer's disease: recommendations from the national institute on aging-Alzheimer's association workgroups on diagnostic guidelines for Alzheimer's disease. Alzheimers Dement 7:263-269.

Okello A, Koivunen J, Edison P, Archer HA, Turkheimer FE, Någren K, Bullock R, Walker Z, Kennedy A, Fox NC, Rossor MN, Rinne JO, Brooks DJ (2009) Conversion of amyloid positive and negative MCI to AD over 3 years: an ${ }^{11} \mathrm{C}-\mathrm{PIB}$ PET study. Neurology 73:754-760.

Owen DR, Yeo AJ, Gunn RN, Song K, Wadsworth G, Lewis A, Rhodes C, Pulford DJ, Bennacef I, Parker CA, StJean PL, Cardon LR, Mooser VE, Matthews PM, Rabiner EA, Rubio JP (2012) An 18-kDa translocator protein (TSPO) polymorphism explains differences in binding affinity of the PET radioligand PBR28. J Cereb Blood Flow Metab 32:1-5.

Passamonti L, Rodríguez PV, Hong YT, Allinson KSJ, Bevan-Jones WR, Williamson D, Jones PS, Arnold R, Borchert RJ, Surendranathan A, Mak E, Su L, Fryer TD, Aigbirhio FI, O’Brien JT, Rowe JB (2018) $\left[{ }^{11} \mathrm{C}\right]$ PK11195 binding in Alzheimer disease and progressive supranuclear palsy. Neurology 90:e1989-e1996.

Petersen RC, Smith GE, Waring SC, Ivnik RJ, Tangalos EG, Kokmen E
(1999) Mild cognitive impairment: clinical characterization and outcome. Arch Neurol 56:303-308.

Satterthwaite TD, Elliott MA, Gerraty RT, Ruparel K, Loughead J, Calkins ME, Eickhoff SB, Hakonarson H, Gur RC, Gur RE, Wolf DH (2013) An improved framework for confound regression and filtering for control of motion artifact in the preprocessing of resting-state functional connectivity data. Neuroimage 64:240-256.

Schuitemaker A, van Berckel BN, Kropholler MA, Veltman DJ, Scheltens P, Jonker C, Lammertsma AA, Boellaard R (2007) SPM analysis of parametric (R)- $\left[{ }^{11} \mathrm{C}\right]$ PK11195 binding images: plasma input versus reference tissue parametric methods. Neuroimage 35:1473-1479.

Shafto MA, Tyler LK, Dixon M, Taylor JR, Rowe JB, Cusack R, Calder AJ, Marslen-Wilson WD, Duncan J, Dalgleish T, Henson RN, Brayne C, Matthews FE; Cam-CAN (2014) The Cambridge Centre for ageing and neuroscience (Cam-CAN) study protocol: a cross-sectional, lifespan, multidisciplinary examination of healthy cognitive ageing. BMC neurology 14:204.

Shirer WR, Ryali S, Rykhlevskaia E, Menon V, Greicius MD (2012) Decoding subject-driven cognitive states with whole-brain connectivity patterns. Cereb Cortex 22:158-165.

Stefanetti V, Beccati F, Passamonti F, Sgariglia E, Coletti M, Vuerich M, Marenzoni ML (2016) Detection and DNA quantification of enterococcus casseliflavus in a foal with septic meningitis. J Am Vet Med Assoc 249:96-100.

Stefaniak J, O’Brien J (2016) Imaging of neuroinflammation in dementia: a review. J Neurol Neurosurg Psychiatry 87:21-28.

Thompson B, Borrello GM (1985) The importance of structure coefficients in regression research. Educ Psychol Meas 45:203-209.

Tofaris GK, Buckley NJ (2018) Convergent molecular defects underpin diverse neurodegenerative diseases. J Neurol Neurosurg Psychiatry 89: 962-969.

Tsvetanov KA, Henson RN, Tyler LK, Razi A, Geerligs L, Ham TE, Rowe JB (2016) Extrinsic and intrinsic brain network connectivity maintains cognition across the lifespan despite accelerated decay of regional brain activation. J Neurosci 36:3115-3126.

Tsvetanov KA, Ye Z, Hughes L, Samu D, Treder MS, Wolpe N, Tyler LK, Rowe JB; Cambridge Centre for Ageing and Neuroscience (2018) Activity and connectivity differences underlying inhibitory control across the adult lifespan. J Neurosci 38:7887-7900.

Turkheimer FE, Edison P, Pavese N, Roncaroli F, Anderson AN, Hammers A, Gerhard A, Hinz R, Tai YF, Brooks DJ (2007) Reference and target region modeling of $\left[{ }^{11} \mathrm{C}\right]-(\mathrm{R})-\mathrm{PK} 11195$ brain studies. J Nucl Med 48:158-167.

Villegas-Llerena C, Phillips A, Garcia-Reitboeck P, Hardy J, Pocock JM (2016) Microglial genes regulating neuroinflammation in the progression of Alzheimer's disease. Curr Opin Neurobiol 36:74-81.

Wang MM, Miao D, Cao XP, Tan L, Tan L (2018) Innate immune activation in Alzheimer's disease. Ann Transl Med 6:177.

Weiler M, Northoff G, Damasceno BP, Balthazar MLF (2016) Self, cortical midline structures and the resting state: implications for Alzheimer's disease. Neurosci Biobehav Rev 68:245-255.

Xu L, Groth KM, Pearlson G, Schretlen DJ, Calhoun VD (2009) Sourcebased morphometry: the use of independent component analysis to identify gray matter differences with application to schizophrenia. Human brain mapping 30:711-724. 